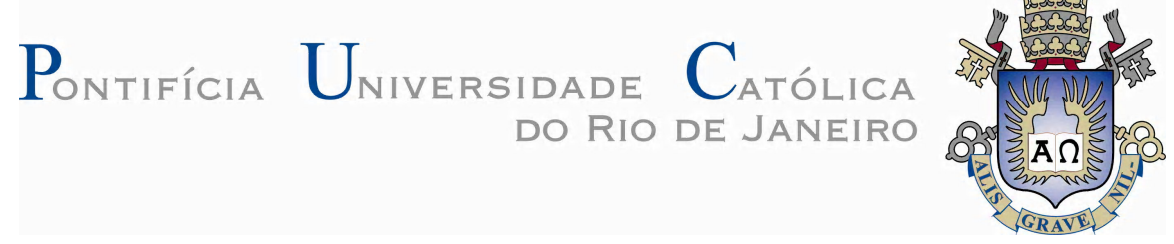

Priscila Mariana da Silva Maia

Fosforimetria na temperatura ambiente em substrato sólido para determinação do cloridrato de irinotecana, e traços do contaminante camptotecina em formulações farmacêuticas anticâncer.

Dissertação de Mestrado

Dissertação apresentada como requisito parcial para obtenção do título de Mestre pelo Programa de PósGraduação em Química da PUC-Rio.

Orientador: Ricardo Queiroz Aucélio

Co-Orientadora: Alessandra Licursi M. C. Cunha

Rio de Janeiro, Março de 2010 


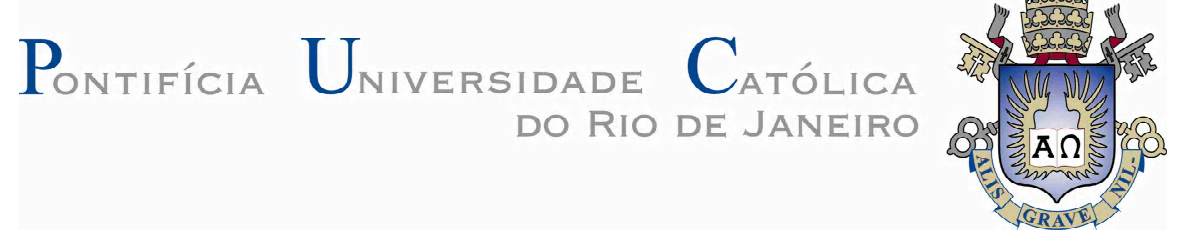

Priscila Mariana da Silva Maia

\title{
Fosforimetria na temperatura ambiente em substrato sólido para determinação do cloridrato de irinotecana, e traços do contaminante camptotecina em formulações farmacêuticas anticâncer.
}

\begin{abstract}
Dissertação apresentada como requisito parcial para obtenção do título de Mestre pelo Programa de Pós-Graduação em Química da PUC-Rio. Aprovada pela Comissão Examinadora abaixo assinada.
\end{abstract}

\author{
Prof. Ricardo Queiroz Aucélio \\ Orientador \\ Departamento de Química - PUC-Rio \\ Prof $^{a}$. Alessandra Licursi M. C. Cunha \\ Co-Orientadora \\ Departamento de Química - PUC-Rio \\ Prof. Ricardo J. Cassella \\ Departamento de Química - UFF \\ Prof $^{\text {a }}$. Fátima Ventura Pereira Meirelles \\ Departamento de Química - PUC-Rio \\ Prof $^{a}$. Tatiana Saint' Pierre \\ Departamento de Química - PUC-Rio
}

Prof. José Eugenio Leal Coordenador Setorial de Pesquisa e Pós-Graduação do Centro Técnico Científico - PUC-Rio

Rio de Janeiro, 19 de março de 2010 
Todos os direitos reservados. É proibida a reprodução total ou parcial do trabalho sem autorização da universidade, da autora e do orientador.

Priscila Mariana da Silva Maia Graduou-se em Farmácia pela Universidade Estácio de Sá em 2007. Estagiou no Laboratório Químico Farmacêutico da Aeronáutica (LAQFA) no ano de 2007.

Ficha Catalográfica

\section{Maia, Priscila Mariana da Silva}

Fosforimetria na temperatura ambiente em substrato sólido para determinação do cloridrato de irinotecana, e traços do contaminante camptotecina em formulações farmacêuticas anticâncer / Priscila Mariana da Silva Maia ; orientador: Ricardo Queiroz Aucélio; co-orientador: Alessandra Licursi M. C. Cunha - 2010.

124 f. ; $30 \mathrm{~cm}$

Dissertação (Mestrado em Química)Pontifícia Universidade Católica do Rio de Janeiro, Departamento de Química, 2010.

Inclui bibliografia

1. Química - Teses. 2. Camptotecina. 3. Irinotecana. 4. Topotecana. 5. Fosforimetria a temperatura ambiente. 6. Incerteza da medição. I. Aucélio, Ricardo Queiroz. II. Pontifícia Universidade Católica do Rio de Janeiro. Departamento de Química. III. Título. 
Acima de tudo, a Deus,

por mais uma conquista.

Aos meus pais, Elvio e Ângela, pela dedicação no meu crescimento e formação.

Em especial, ao meu marido Sandro, pelo incentivo, paciência e companheirismo. Amo você! 


\section{Agradecimentos}

Ao Professor e orientador Ricardo Queiroz Aucélio, pela oportunidade, confiança, incentivo, paciência e pela orientação durante a realização deste trabalho. Muito obrigada!

Em especial a Co-orientadora Alessandra Licursi pelo seu grande ensinamento, incentivo, apoio e companheirismo, que inúmeras vezes me encaminhou para tomadas de decisões importantes.

A todos os professores do Departamento de Química da PUC-Rio, os quais muito contribuíram para o aumento do meu conhecimento.

À equipe do LEEA, Eliane, Cabrini, Elaine, Flávia, Sônia, Paulo, Thiago pelo apoio durante esse trabalho.

Aos professores participantes da comissão examinadora.

Aos funcionários do Departamento de Química da PUC-Rio, em especial a Fátima pela ajuda durante todo o período de estudo.

À PUC-Rio pela organização e qualidade do curso oferecido.

Ao CNPq pela bolsa de estudo fornecida.

A todos aqueles que de alguma forma contribuíram para que este trabalho fosse realizado. 


\section{Resumo}

Maia, Priscila Mariana da Silva; Aucélio, Ricardo Queiroz. Fosforimetria na temperatura ambiente em substrato sólido para determinação do cloridrato de irinotecana, princípio ativo do anticâncer injetável, e traços do contaminante camptotecina em formulações farmacêuticas anticâncer. Rio de Janeiro, 2010. 124p. Dissertação de Mestrado Departamento de Química, Pontifícia Universidade Católica do Rio de Janeiro.

Os derivados da camptotecina (CPT), irinotecana (CPT-11) e topotecana (TPT) são usados para o tratamento do câncer, sendo a CPT um potencial contaminante em medicamentos anticâncer a base de CPT-11 ou TPT. Neste trabalho, a fosforimetria na temperatura ambiente em substrato sólido (SSRTP) foi proposta como técnica analítica para a determinação do princípio ativo do anticâncer injetável a base de CPT-11 e de traços do contaminante CPT em formulações farmacêuticas anticâncer. As características fosforescentes dos dois analitos foram estudadas de modo univariado em função de diversos parâmetros experimentais, como o tipo e a quantidade de sal de átomo pesado indutor de fosforescência, influência do valor do pH do tampão usado na solução do analito e quantidade de surfactante modificador de superfície da celulose. Uma vez definidos os fatores relevantes, o planejamento fatorial do tipo composto central foi realizado para a determinação de traços do contaminante (CPT) com o intuito de estudar os efeitos principais e as possíveis interações entre os fatores de resposta visando à escolha da melhor condição experimental. As melhores condições foram obtidas usando substratos de celulose contendo $332 \mu \mathrm{g}$ de $\mathrm{TINO}_{3}$ (indutor da fosforescência) em solução carreadora contendo tampão Britton-Robinson ( $\mathrm{pH}$ 10,5). Para a CPT-11 a otimização foi realizada apenas pela abordagem univariada, com as seguintes condições escolhidas: $662 \mu \mathrm{g}$ de $\mathrm{Pb}\left(\mathrm{NO}_{3}\right)_{2}$ em substratos de celulose contendo $577 \mu \mathrm{g}$ de SDS. A CPT pôde ser determinada seletivamente em matrizes contendo 40 vezes mais TPT, em concentração, usando a determinação no ponto isodiferencial $(367 \mathrm{~nm})$ da derivada de $2^{-}$ordem do espectro de excitação. Para matrizes contendo CPT-11, esse desempenho foi mais limitado, pois a CPT só foi determinada seletivamente em misturas contendo até 5 vezes mais CPT-11. Para cada uma das condições selecionadas foram realizados estudos para obtenção dos parâmetros de desempenho. Em ambos os casos, a resposta analítica teve comportamento linear (homocedático) em função da massa de CPT ou de CPT-11 presentes no substrato de celulose. Os limites de detecção e de quantificação absolutos 
ficaram na ordem do ng. Um estudo detalhado da estimativa da incerteza de medição também foi realizado e a incerteza combinada associada à medição de fosforescência da CPT foi de até $16 \%$. O método foi aplicado na quantificação de CPT-11 em soluções injetáveis com $97,5 \pm 5,5 \%$ de recuperação e na determinação de CPT em medicamentos a base de TPT $(101,5 \pm 3,5 \%$ de recuperação medindo em $367 \mathrm{~nm}$ do espectro de excitação após derivação de $2^{\mathrm{a}}$ ordem) e nas matrizes urina (102,5 $\pm 3,5 \%$ de recuperação) e saliva (102,5 \pm $4,5 \%$ de recuperação), ambas fortificadas com CPT e usando-se detecção em $570 \mathrm{~nm}$ do espectro de emissão de varredura normal. Testes comparativos entre a SSRTP e a HPLC-DF foram realizados e os resultados foram satisfatórios para um nível de 95\% de confiança. Uma comparação entre diferentes substratos foi também realizada para avaliar requisitos práticos, variabilidade de sinal do analito e do branco, o que indicou vantagens do substrato de nylon sobre o de celulose.

\section{Palavras-chave}

Camptotecina; irinotecana; topotecana; fosforimetria na temperatura ambiente; incerteza da medição. 


\section{Abstract}

Maia, Priscila Mariana da Silva; Aucélio, Ricardo Queiroz (Advisor). Solid substrate room-temperature phosphorimetry for the irinotecan hydrochloride determination, active principle of injectable anti-cancer drugs, and traces of contaminants in pharmaceutical formulations camptothecin anti-cancer. Rio de Janeiro, 2010. 124p. MSc. Dissertation - Departamento de Química, Pontifícia Universidade Católica do Rio de Janeiro.

Irinotecan (CPT-11) and topotecan (TPT) are employed for cancer treatment and camptothecin (CPT) is a potential contaminant in anti-cancer drugs based on CPT-11 or TPT. In this work, solid substrate room-temperature phosphorimetry (SSRTP) was proposed as analytical technique for the quantification of CPT-11 in anti-cancer drugs and for the determination of traces of CPT in CPT-11 and TPT based anti-cancer pharmaceutical formulations. The phosphorescence characteristics of the analytes have been studied and experimental conditions (type and amount of the heavy atom salts used to induce phosphorescence, influence of the $\mathrm{pH}$ of the analyte carrier solution and the amount of surface modifier) were optimized in an univariate way. For the method aiming the determination of CPT, a further optimization using a central composite design was made in order to identify the main effects and possible interactions among factors. The best conditions had been achieved using cellulose substrate containing $332 \mu \mathrm{g}$ of $\mathrm{TINO}_{3}$ (phosphorescence inducer) and analyte carrier solution containing Britton-Robinson buffer ( $\mathrm{pH}$ 10.5). For the CPT-11, best conditions were achieved in cellulose substrates containing $577 \mu \mathrm{g}$ of SDS and $662 \mu \mathrm{g}$ of $\mathrm{Pb}\left(\mathrm{NO}_{3}\right)_{2}$ The selective determination of $\mathrm{CPT}$ could be performed in samples containing a higher amount of TPT (40 times) if the signal measurement is made at the isodifferential wavelength $(367 \mathrm{~nm})$ of the $2^{\text {nd }}$ derivative excitation spectra. For samples containing CPT-11, selective determination of CPT could be made in samples containing CPT-11/CPT molar proportion no higher than 5 . Parameters of merit have been obtained for both methods. Analytical responses presented linear behavior in the in working range from the limit of quantification up to at least $348.0 \mathrm{ng}$ of CPT or $440.2 \mathrm{ng}$ of CPT-11 (deposited in the center of the substrate). Absolute limits of detection and quantification were 26.8 and 42.3 ng for CPT and 79.6 and 99.9 ng for CPT-11. A detailed metrological study was performed for the measurement of CPT and the combined uncertainty associated to the phosphorescence measurement was $16 \%$. The method was applied for the quantification of CPT-11 in injectable solutions with recovery of $97.5 \pm 5.5 \%$. For CPT, recovery in TPT based pharmaceutical formulation, previously fortified with 
the analyte, was $101.5 \pm 3.5 \%$ (measurement made at $367 \mathrm{~nm}$ of the $2^{\text {nd }}$ derivative excitation spectra). In analyte fortified urine and saliva, recoveries were respectively $102.5 \pm 3.5 \%$ and $102.5 \pm 4.5 \%$ (using non-derived spectra and detention at $570 \mathrm{~nm}$ of the emission band). Comparative tests between the SSRTP and HPLC-DF have been made and the results agreed (at a 95\% confidence level). A comparison using different substrates (nylon and cellulose) was also performed in order to evaluate practical aspects, analyte signal intensity and the variability of the analyte and blank signals. The result indicated advantages in using nylon substrates for the phosphorimetric determination of CPT.

\section{Keywords}

Camptothecin; irinotecan; topotecan; room-temperature phosphorimetry; measurement uncertainty. 


\section{Sumário}

1 Introdução 20

1.1. Câncer 20

1.2. Medicamentos falsificados $\quad 21$

1.3. Camptotecina e seus derivados 23

1.4. Métodos utilizados na determinação de CPT, CPT-11 e TPT 26

1.5. Fosforescência na temperatura ambiente 29

1.5.1. Fosforimetria em temperatura ambiente e em substrato sólido $\begin{array}{ll}\text { (SSRTP) } & 31\end{array}$

1.5.2. Parâmetros que afetam a intensidade da fosforescência 33

1.5.2.1. Influência do oxigênio e da umidade 33

1.5.2.2. Efeito do átomo pesado 34

1.5.2.3. Efeito do surfactante como modificador de superfície 35

1.5.2.4. Influência do sistema de solventes 35

1.5.2.5. Influência do $\mathrm{pH} \quad 36$

1.5.3. Técnica para o aumento da seletividade 36

1.6. Objetivos 38

1.6.1. Objetivo geral 38

1.6.2. Objetivos específicos 38

2 Instrumentação, materiais e métodos $\quad 39$

2.1. Instrumentação 39

2.1.1. Sistema de lavagem e secagem dos substratos 39

2.1.2. Reator fotoquímico 40

2.1.3. Espectrofotômetro de luminescência 40

2.1.4. Cromatógrafo em fase líquida de alta eficiência 42

2.1.5. Outros equipamentos auxiliares 42

2.2. Reagentes, soluções e materiais 43

2.3. Procedimento geral 44

2.3.1. Procedimento geral para medição da fosforescência 45

3 Resultados e discussão - características fosforescentes dos anticancerígenos e otimizações $\quad 47$

3.1. Estudos preliminares dos anticancerígenos 47 
3.1.1. Efeito externo do átomo pesado 50

3.1.2. Efeito do SDS 51

3.1.3. Estudo da influência do tratamento fotoquímico (radiação UV) 52

3.1.4. Considerações sobre o estudo preliminar 52

3.2. Maximização da fosforescência $\quad 55$

3.2.1. Estudos univariados com a CPT 55

3.2.1.1. Solubilidade $\quad 55$

3.2.1.2. $\mathrm{pH}$ da solução carreadora de analito 56

3.2.1.3. Massa de sal de átomo pesado no substrato 57

3.2.1.4. Presença de SDS no substrato de celulose 58

3.2.2. Estudo multivariado (planejamento fatorial composto central) para a $\begin{array}{ll}\text { CPT } & 59\end{array}$

3.2.3. Estudos univariados com a CPT-11 63

3.2.4. Otimização de parâmetros instrumentais 65

3.2.4.1. Tempo de atraso - Delay 65

$\begin{array}{ll}\text { 3.2.4.2. Banda espectral de passagem } & 67\end{array}$

3.2.5. Condições experimentais e instrumentais otimizadas 68

3.3. Comparação entre diferentes substratos (nylon e celulose) 71

4 Resultados e discussão - estudos de seletividade e validação 74

4.1. Seletividade $\quad 74$

4.1.1. Avaliação da interferência dos alcalóides $\quad 74$

4.1.2. Avaliação da interferência de fluidos biológicos na fosforescência da CPT $\quad 78$

4.2. Validação dos métodos $\quad 80$

4.2.1. Faixa de resposta linear $\quad 80$

4.2.2. Detectabilidade 86

$\begin{array}{ll}\text { 4.2.3. Robustez } & 88\end{array}$

4.2.4. Precisão $\quad 89$

4.2.5. Incerteza de medição de fosforescência 91

4.3. Aplicação do método 101

4.3.1. Recuperação em amostras 101

4.3.2. Comparação com HPLC 103

5 Conclusão e trabalhos futuros 106

6 Referências 109 
7 Anexo 


\section{Lista de tabelas}

Tabela 1: Métodos analíticos por HPLC para a determinação de camptotecina e seus derivados

Tabela 1 (continuação): Métodos analíticos por HPLC para a determinação de camptotecina e seus derivados

Tabela 2: Estudo do efeito de sais de átomos pesados no sinal fosforescente da irinotecana (CPT-11), camptotecina (CPT) e topotecana (TPT) em substrato de celulose ${ }^{a}$.

Tabela 3: Estudo do efeito de sais de átomos pesados no sinal fosforescente da irinotecana (CPT-11), camptotecina (CPT) e topotecana (TPT) em substrato de celulose na presença de SDS (360 $\mu \mathrm{g})^{\mathrm{a}}$.

Tabela 4: Resultados obtidos com os experimentos do planejamento fatorial composto central $2^{2}$ usando $\mathrm{TINO}_{3}$ como sal indutor de sinal fosforescente para a CPT (111 ng).

Tabela 5: Condições experimentais e instrumentais escolhidas para determinação fosforimétrica da CPT e CPT-11.

Tabela 6: Avaliação da interferência do CPT-11 e do TPT na fosforescência do CPT com medições a $570 \mathrm{~nm}$ do espectro de emissão obtido por varredura normal.

Tabela 7: Estudo de interferência da fosforescência da CPT em misturas contendo CPT/TPT.

Tabela 8: Parâmetros analíticos para a CPT, CPT usando varredura de $2^{\underline{a}}$ ordem, CPT depositada no substrato de nylon e para a CPT-11.

Tabela 9: Dados da capacidade de detecção da CPT depositada em substratos de celulose nos espectros de varredura normal $(\mathrm{N})$ e derivada de $2^{\mathrm{a}}$ ordem (D) e no substrato de nylon

Tabela 10: Dados da capacidade de detecção da CPT-11

Tabela 11: Avaliação da Robustez para o CPT e CPT-11.

Tabela 12: Valores do CV (\%) nos três pontos da curva analítica da CPT depositada em substratos de celulose (espectros de varredura normal, $\mathrm{N}$ e varredura de $2^{\mathrm{a}}$ derivada, D) e depositada em substratos de nylon.

Tabela 13: Valores do CV (\%) nos três pontos da curva analítica da CPT- 
11 depositada em substratos de celulose.

Tabela 14: Resultados de incertezas da medição da fosforescência da CPT em substratos de celulose em diferentes concentrações: $2,4 \times 10^{-5} \mathrm{~mol}$ $\mathrm{L}^{-1}(41,8 \mathrm{ng}) ; 4,8 \times 10^{-5} \mathrm{~mol} \mathrm{~L}^{-1}(83,6 \mathrm{ng})$ e $7,4 \times 10^{-5} \mathrm{~mol} \mathrm{~L}^{-1}(128,9 \mathrm{ng})$ nos espectros de varredura normal. Os valores da obtidos pela $2^{\underline{a}}$ derivada encontram-se dentro dos parênteses.

Tabela 15: Resultados de incerteza da medição da fosforescência do CPT em substrato de nylon com diferentes concentrações: $9,0 \times 10^{-6} \mathrm{~mol} \mathrm{~L}$ ${ }^{1}$ (15,7 ng); 3,2 × 10 $0^{-5} \mathrm{~mol} \mathrm{~L}^{-1}$ (55,7 ng) e $5,5 \times 10^{-5} \mathrm{~mol} \mathrm{~L}^{-1}$ (95,8 ng).

Tabela 16: Resultados de incerteza da medição da fosforescência do CPT11 em diferentes concentrações: $3,0 \times 10^{-5} \mathrm{~mol} \mathrm{~L}^{-1}(101,6 \mathrm{ng}) ; 4,5 \times 10^{-}$

${ }^{5} \mathrm{~mol} \mathrm{~L}^{-1}(152,4 \mathrm{ng})$ e $7,5 \times 10^{-5} \mathrm{~mol} \mathrm{~L}^{-1}(253,9 \mathrm{ng})$

Tabela 17: Resultado do teste da comparação entre SSRTP e HPLC para a CPT.

Tabela 18: Resultado do teste da comparação entre SSRTP e HPLC para a CPT-11.

Tabela 19: Comparação entre os métodos de análise desenvolvidos. (+++) Melhor avaliação. (++) Avaliação intermediária. (+) Pior avaliação. 


\section{Lista de figuras}

Figura 1: Mecanismo de ação da camptotecina.

Figura 2: Estrutura química da camptotecina, da irinotecana e da topotecana.

Figura 3: Esquema eletrônico para o estado fundamental e para as duas configurações do estado excitado de menor energia.

Figura 4: Diagrama modificado de Jablonskii. 31

Figura 5: Sistema usado para lavagem dos papéis. 39

Figura 6: Secagem do substrato de papel. 39

Figura 7: Reator fotoquímico com seis lâmpadas de mercúrio. 40

Figura 8: Esquema óptico do espectrofotômetro de luminescência LS 55 Perkin Elmer.

Figura 9: Aparato de medição em superfície sólida.

Figura 10: Sistema de purga com nitrogênio, composta por unidade de desoxigenação e de desumidificação do gás.

Figura 11: Planilha para auxiliar na aplicação da amostra.

Figura 12: Dessecador sob efeito do vácuo e protegido da luz.

Figura 13: Espectros dos três alcalóides $\left(4 \times 10^{-4} \mathrm{~mol} \mathrm{~L}^{-1}\right)$ na presença do AP de $\mathrm{TI}(\mathrm{I})(332 \mu \mathrm{g})$.

Figura 14: Espectros dos três alcalóides $\left(4 \times 10^{-4} \mathrm{~mol} \mathrm{~L}^{-1}\right)$ na presença $\mathrm{Pb}(\mathrm{II})(414 \mu \mathrm{g})$ em substratos contendo SDS(360 $\mu \mathrm{g})$.

Figura 15: Influência da proporção de metanol no sistema de solventes da solução carreadora na fosforescência da CPT (111 ng) em substratos de celulose contendo nitrato de tálio $(332 \mu \mathrm{g})$.

Figura 16: Influência da variação do $\mathrm{pH}$ na fosforescência o sinal fosforescente da CPT (111 ng) em substratos de celulose contendo nitrato de tálio $(332 \mu \mathrm{g})$.

Figura 17: Estudo do efeito da massa de $\mathrm{TINO}_{3}$ presente no substrato de celulose na fosforescência da CPT (111 ng).

Figura 18: Gráfico de Pareto para fosforescência da CPT (111 ng) induzida por $\mathrm{TINO}_{3}$.

Figura 19: Curva de nível obtida no planejamento fatorial da CPT (111 ng) na presença de $\mathrm{TI}(\mathrm{I})$.

Figura 20: Curva univariada da variação de fosforescência da CPT (111 
ng) em função da massa de $\mathrm{TINO}_{3}$ depositada no substrato.

Figura 21: Estudo do efeito da massa de $\mathrm{Pb}\left(\mathrm{NO}_{3}\right)_{2}$ presente no substrato de celulose contendo SDS $(360 \mu \mathrm{g})$ na fosforescência da CPT-11(135 $\mathrm{ng})$.

Figura 22: Estudo do efeito do SDS presente no substrato de celulose na fosforescência do CPT-11 (135 ng) na presença do $\mathrm{Pb}\left(\mathrm{NO}_{3}\right)_{2}$ (662 $\mu \mathrm{g})$.

Figura 23: Influência do tempo de atraso de detecção na fosforescência da CPT (696 ng) em substrato de papel contendo $332 \mu \mathrm{g}$ de $\mathrm{TINO}_{3}$.

Figura 24: Influência do tempo de atraso de detecção na fosforescência da CPT-11 (101 ng) em substrato de papel contendo $577 \mu \mathrm{g}$ de SDS e $662 \mu \mathrm{g}$ de $\mathrm{Pb}\left(\mathrm{NO}_{3}\right)_{2}$.

Figura 25: Estudo da influência da banda espectral de passagem de emissão na fosforescência da CPT (696 ng) em substrato de celulose contendo $332 \mu \mathrm{g}$ de $\mathrm{TINO}_{3}$.

Figura 26: Estudo da influência da banda espectral de passagem de emissão na fosforescência da CPT-11 (101 ng) em substrato de celulose contendo $577 \mu \mathrm{g}$ de SDS e $662 \mu \mathrm{g}$ de $\mathrm{Pb}\left(\mathrm{NO}_{3}\right)_{2}$.

Figura 27: Espectro de fosforescência da CPT (348 ng) (a) antes da otimização e (b) depois da otimização experimental e instrumental.

Figura 28: Espectro de fosforescência da CPT-11 (135 ng) (a) antes da otimização e (b) depois da otimização experimental e instrumental.

Figura 29: Espectros de fosforescência da CPT (69 ng) em diferentes substratos (a) celulose (b) nylon ( sal de AP: $332 \mu \mathrm{g}$ de $\mathrm{TINO}_{3}$ ).

Figura 30: Espectros dos ensaios em branco do substrato de celulose e nylon

Figura 31: Estudo de interferência da CPT-11 no sinal fosforescente da CPT.

Figura 32: Espectros da derivada de $2^{\underline{a}}$ ordem da CPT em soluções contendo concentrações de TPT 40 vezes maior.

Figura 33: Brancos da excitação (a) antes e (b) depois da derivação.

Figura 34: Espectros de emissão da recuperação da CPT $\left(4 \times 10^{-4} \mathrm{~mol} \mathrm{~L}^{-1}\right)$ em urina.

Figura 35: Espectros de emissão da recuperação da CPT $\left(4 \times 10^{-4} \mathrm{~mol} \mathrm{~L}^{-1}\right)$ em saliva.

Figura 36: Curvas analíticas da CPT (a) varredura normal e (b) varredura derivada de $2^{\mathrm{a}}$ ordem. 
Figura 37: Gráficos de resíduos da CPT (a) varredura normal e (b) varredura derivada de $2^{\mathrm{a}}$ ordem.

Figura 38: Curva analítica da CPT depositada no substrato de nylon. $\quad 84$

Figura 39: Gráfico de resíduo da CPT depositada no substrato de nylon. $\quad 84$

Figura 40: Curva analítica da CPT-11 em substrato de celulose. 85

Figura 41: Gráfico de resíduo da CPT-11. 85

Figura 42: Diagrama de causa e efeito para medição de fosforescência por SSRTP $^{78}$.

Figura 43:Gráficos de contribuição das fontes de incerteza da CPT em diferentes concentrações no substrato de celulose: (a) $2,4 \times 10^{-5}$, (b) $4,8 \times 10^{-5}$ e (c) $7,4 \times 10^{-5} \mathrm{~mol} \mathrm{~L}^{-1}$

Figura 44: Gráficos de contribuição das fontes de incerteza da CPT em diferentes concentrações no substrato de nylon: (a) $9,0 \times 10^{-6}$ (b) $3,2 \times$ $10^{-5}$ (c) $5,5 \times 10^{-5} \mathrm{~mol} \mathrm{~L}^{-1}$

Figura 45: Gráficos de contribuição das fontes de incerteza da CPT-11 em diferentes concentrações: (a) $3,0 \times 10^{-5}$ (b) $4,5 \times 10^{-5}$ (c) $7,5 \times 10^{-5} \mathrm{~mol}$ $\mathrm{L}^{-1}$

Figura 46: Espectros de emissão da recuperação da CPT $\left(4 \times 10^{-4} \mathrm{~mol} \mathrm{~L}^{-1}\right)$ em formulações farmacêuticas (CPT-11 e TPT).

Figura 47: Espectros de emissão da recuperação da CPT-11 em medicamento. 


\section{Siglas e abreviações}

ABNT - Associação brasileira de normas técnicas

ANOVA - Análise de variância

ANVISA - Agência nacional de vigilância sanitária

AP - Átomo pesado

$\mathrm{Cl}$ - Cruzamento interno

CIS - Cruzamento intersistemas

CPT - Camptotecina

CPT-11 - Irinotecana

CV - Coeficiente(s) de variação

Em - Emissão

ESI-MS - Eletrospray- espectrometria de massas

ETCO - Instituto Brasileiro de Ética Concorrencial

Ex - Excitação

HPLC - Cromatografia líquida de alta eficiência

$\mathrm{I}_{\mathrm{A}}$ - Intensidade fosforescente do analito

$I_{A}-I_{B}-$ Sinal fosforescente líquido

$I_{B}$ - Intensidade fosforescente do branco

$\mathrm{I}_{\mathrm{CPT}}$ - Intensidade de sinal fosforescente da CPT

$\mathrm{I}_{\mathrm{CPT}-11}$ - Intensidade de sinal fosforescente de misturas contendo CPT +

CPT-11

INMETRO - Instituto nacional de metrologia

ISP-MS - íon spray espectrometria de massas

$\mathrm{I}_{\text {TPT }}$ - Intensidade de sinal fosforescente de misturas contendo CPT + TPT

LC - cromatografia líquida

LD - Limite de detecção

LDA - Limite de detecção absoluto

LEEA - Laboratório de Espectroanalítica e Eletroanalítica Aplicada

LQ - Limite de quantificação

LQA - Limite de quantificação absoluto

MEKC - Eletroforese capilar com uso de meio micelar

MS - Espectrometria de massa

OMS - Organização Mundial de Saúde 
PF- Planejamento fatorial

RSD - Desvio(s) padrão relativo(s)

$\mathrm{RV}$ - Relaxamento vibracional

$\mathrm{S}_{0}$ - Estado fundamental singleto

$\mathrm{S}_{1}$ - Estado excitado singleto

SDS - Dodecil sulfato de sódio

SPE- Solid phase extraction

$\mathrm{S}_{\mathrm{n}}$ - Estado excitado singleto de maior energia

SSRTP - Fosforimetria em temperatura ambiente suportada em substrato

sólido

$\mathrm{T}_{0}$ - Estado fundamental tripleto

$\mathrm{T}_{1}$ - Estado excitado tripleto

$\mathrm{T}_{\mathrm{n}}$ - Estado excitado tripleto de maior energia

TPT - Topotecana

u.a. - Unidades arbitrárias

US FDA - United States Food e Drug Administration

UV - ultravioleta

$\lambda_{\mathrm{em}}$ - Comprimento de onda de emissão expresso em nm

$\lambda_{\text {exc }}$ - Comprimento de onda de excitação expresso em $\mathrm{nm}$

$\lambda_{\text {iso }}$ - Comprimento de onda isodiferencial 